\title{
Nadie se queda atrás: la integración del alumnado de E/LE en el aula de secundaria
}

SARA BADIA-CLIMENT

Universitat de València

sara.badia@uv.es

Resumen: En la actualidad, las aulas de secundaria se han convertido en espacios multiculturales que han llevado tanto a alumnos como a profesores a enfrentarse a una nueva realidad dentro de las clases. Los alumnos de origen extranjero y con lengua no nativa española se ven obligados a adaptarse e integrarse a un ritmo vertiginoso que, en ciertas circunstancias, es inasumible; especialmente en aquellos alumnos que han entrado dentro del circuito escolar a partir del ciclo de secundaria y en mitad de curso.

El objetivo, por tanto, de este artículo es analizar los resultados de aprendizaje de los alumnos inmigrantes de $3 .^{\circ}$ de ESO dentro de una secuencia didáctica dedicada al género anuncio para a) detectar la problemática de aprender una lengua mientras se tratan de impartir los contenidos curriculares correspondientes $\mathrm{y}$, sobre ellos, b) proponer una serie de modificaciones que puedan paliar estas dificultades.

Palabras clave: Análisis de Errores, Enfoque por tareas, Enfoque Comunicativo, ELE en Secundaria,

\section{No one is left behind: integration of E/LE high school students}

Abstract: Nowadays, secondary classrooms have turned into multicultural spaces that have driven students and teachers to a new academic reality. Foreign students that haven't got Spanish as native language must adapt and include in a fast-way. This situation, sometimes, is unacceptable, especially when students have started the scholarcircuit in the middle of course.

For this reason, the aim of this paper is to analyse the learning results of non-native students of 3.tr of ESO during a didactic sequence of advertisement genre to a) detect the troubles of learning a new language while they are trying to assimilate the curricular contents and, therefore, b) propose a series of modifications that could palliate these difficulties.

Key words: Mistake's Analysis, Tasks-Based Approach, Communicative Approach, Spanish as foreign language in High Schools

\section{Introducción}

La realidad de las aulas de secundaria cada vez se va volviendo más compleja y heterogénea debido, por un lado, al aumento de la ratio de estudiantes y, por otro, a su distinta procedencia. Esta circunstancia que, si bien multiplica las posibilidades y enriquece el espacio de aprendizaje, obliga al docente a repensar y adecuar tanto los contenidos como la metodología para que todo el grupo de clase pueda seguir el ritmo y sea capaz de conseguir, en definitiva, los objetivos que plantea el currículum. 
Por esta razón, es posible que la implementación de una secuencia didáctica no obtenga los resultados esperados en todo el conjunto del alumnado, especialmente en aquellos que no tienen un dominio suficiente de la lengua vehicular para seguir el hilo de las clases. En consecuencia, esta circunstancia lleva a que sea necesario analizar los pasos y los resultados con el fin de mejorar los contenidos en una actuación posterior. En este trabajo, en concreto, presentamos esta circunstancia: una secuencia didáctica sobre contenidos publicitarios (concretamente, el género anuncio) que se desarrolló teniendo en cuenta una serie de objetivos que, posteriormente, no dieron los frutos deseados debido a la diversidad/variedad/pluralidad del alumnado respecto a los diferentes países de origen y al diferente dominio de la lengua meta.

Debido a que los resultados no fueron los esperados y siguiendo la premisa de que hay que repensar los contenidos para mejorar su adaptación y el acceso a ellos de todo el alumnado; los objetivos de este trabajo se dividen en dos: 1) realizar un análisis de errores de la secuencia didáctica planteada, teniendo en cuenta la perspectiva de los alumnos no nativos y, sobre este, 2) realizar una propuesta de mejora que permita acercar los contenidos tratados a todo el conjunto de clase.

Para ello, el artículo se estructura en 4 partes: en primer lugar, se trazan las bases sobre las que se han detectado los errores en el planteamiento e implementación posterior de esta secuencia a partir del respaldo de una bibliografía teórica sobre el tema $(\S 2)$. En base a estos postulados, en segundo lugar, se tratarán los pilares sobre los que se ha fundamentado la nueva propuesta $(\S 3)$. En tercer lugar, se presenta brevemente el contenido y la organización de la secuencia didáctica que se implementó; sobre la que se realiza, junto con los materiales de los alumnos, un análisis de los problemas y errores más frecuentes $(\S 4)$. Finalmente, se expone una propuesta de mejora en la que se pretenden corregir los aspectos reseñados en el análisis anterior (§ 5).

\section{Las aulas de secundaria como punto de encuentro}

Partiendo de la base de que los alumnos tanto nativos como no nativos no son expertos en el dominio de la lengua castellana, es cierto que se puede observar cierto desnivel entre los estudiantes escolarizados en España desde hace años (sean españoles o no) y aquellos que, además de no tener como L1 el español, han sido escolarizados en otros sistemas (o ni siquiera lo han sido). La clase, en esta situación, se convierte en una amalgama de estudiantes con necesidades muy dispares, pero con un objetivo común: adquirir los conocimientos postulados para su nivel de escolarización.

En este sentido, el propio currículum de secundaria contempla que el sistema educativo debe servir para «...promover una sociedad libre, tolerante y justa y que contribuye a defender los valores y principios de la libertad, el pluralismo, ...» (Ley Orgánica 8, 2013: 10), así como educar en valores sociales que ayuden a «...superar cualquier discriminación y la accesibilidad universal a la educación, y que actúe como elemento compensador de las desigualdades personales, culturales, económicas y sociales...» (ibid.: 11).

Por tanto, todo estudiante que ingrese en el circuito educativo debe contar con el apoyo y las posibilidades suficientes para que se desarrolle plenamente como ciudadano. No obstante, la realidad que se experimenta en ciertos contextos dista mucho de la deseada, ya que existe una falta de materiales y de atención específica al alumnado no nativo en 
las clases de ESO, debido, entre otros factores, a la falta de recursos, ratios de alumnos desproporcionadas o la carencia de formación del profesorado entre otras cuestiones (Roca Marín, 2002: 6; Morales Orozco, 2006: 27).

Esta circunstancia puede traer como consecuencia la falta de motivación de los estudiantes que no tienen un dominio nativo de la lengua vehicular y que, finalmente, se ve reflejado en mayores tasas de absentismo (Márquez Vázquez y Gualda Caballero, 2013/2014: 64) o mayores índices de fracaso escolar (ver Gráfico 1).

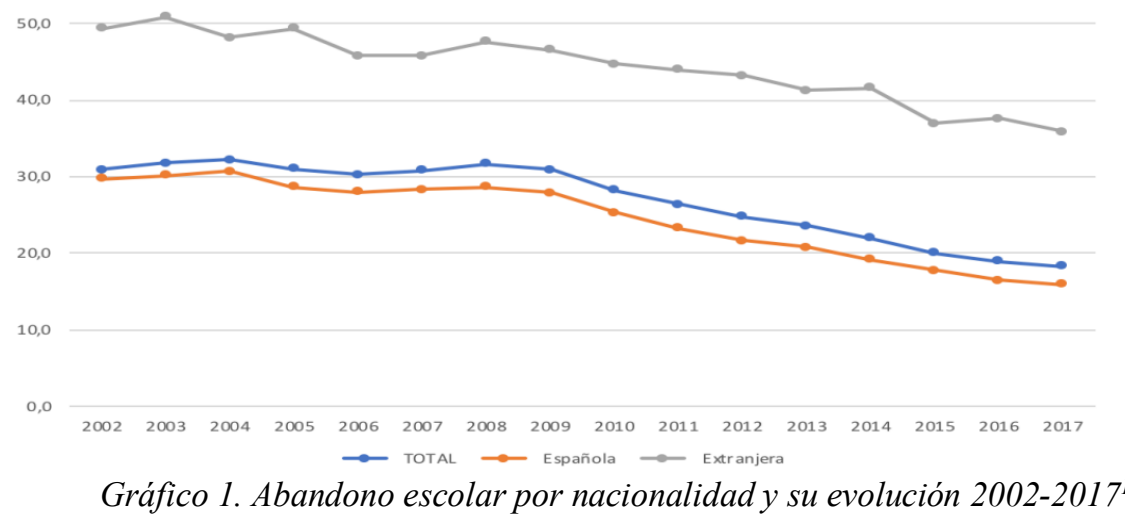

Cabe destacar que la Comunidad Valenciana dispuso el PASE (Programa de Acogida al Sistema Educativo) para combatir esta clase de situaciones y que tiene como objetivo último facilitar a los alumnos no nativos la incorporación a la secundaria. Este programa dota a estos estudiantes de una serie de horas de aprendizaje específico de las lenguas vehiculares de la comunidad o del centro. La contrapartida, sin embargo, reside en que deben hacerse en horario lectivo, restando, de esta forma, tiempo de las clases del resto de asignaturas obligatorias. La conclusión, por tanto, es que se consigue una atención más individualizada para el aprendizaje de E/LE, pero pierden los contenidos que se están impartiendo en el resto de asignaturas que coincidan. Todo ello sin olvidar que no todos los centros cuentan con este programa (41 centros de secundaria en la Comunidad Valenciana en 2013 según datos de la Generalitat Valenciana). Más tarde se ha integrado el programa PAM (Plan de Actuación para la Mejora) que dotaba a los centros con subvenciones para organizar actividades extraescolares, entre las que se encontraba una partida específica para las clases de E/LE. No obstante, de nuevo, el problema reside en que no todos los centros acceden a esta clase de ayudas (355 institutos de secundaria en la Comunidad Valenciana en 2019).

Por lo tanto, es necesario que los profesores (y, probablemente, con más motivo los de Lengua castellana y literatura) sepan modular sus materiales e integrar a aquellos estudiantes que cuenten con dificultades para seguir sus clases. A continuación, se ofrecen unas breves pautas sobre las que se basó la secuencia que se implementó en el aula y sobre la que se ha hecho el posterior análisis de errores y la propuesta de mejora.

\footnotetext{
${ }^{1}$ Fuentes: Federación de Enseñanza de CCOO (2018) y Ministerio de Educación y FP.
} 


\subsection{El currículum, el enfoque comunicativo y el enfoque por tareas}

La etapa de Educación Secundaria Obligatoria tiene como finalidad desarrollar y potenciar las competencias comunicativas de los alumnos, entre otros objetivos. Así se refleja en el Real Decreto 1105/2014, en el que se señala que se debe potenciar la capacidad para «Comprender y expresar con corrección, oralmente y por escrito, en la lengua castellana [...] textos y mensajes complejos...» (ibíd.: 177). De forma concreta, la asignatura de Lengua castellana y literatura tiene el fin de desarrollarla y fomentar la comunicación lingüística (Real Decreto 1105/2014: 357).

Asimismo, el texto legal de la Generalitat Valenciana (Decreto 87/2015) fundamenta que la preparación del alumnado y la metodología de las clases debe seguir el enfoque comunicativo que se defiende en los textos legales (Decreto 87/2015: 3), ya que resulta indispensable para desarrollar las competencias vinculadas a cualquier tipo de expresión la práctica y aplicación. Por ello, se explica que estas tareas deben plantearse con una contextualización adecuada y fomentar el desarrollo de estrategias para analizar, planificar y utilizar el pensamiento crítico (ibid.: 3).

En palabras de Hymes (1972, 1989, 1992), la competencia comunicativa es aquella que nos permite adaptar nuestro discurso a la situación correspondiente; competencia que se adquiere, en gran medida, por la participación sucesiva en contextos comunicativos reales. Esta, según Canale y Swain (1980), se divide en tres competencias que atañen a tres aspectos: 1) el gramatical, es decir, a los elementos vinculados con las reglas morfológicas, sintácticas y semánticas que se inscriben en el nivel de la oración; al 2) pragmático-discursivo, en el que se debe tener en cuenta el contexto de uso y sus normas sociales; y 3) al estratégico, referido a todas aquellas herramientas e instrumentos destinados a «compensar las dificultades en la comunicación debidas a variables de actuación o a actuación insuficiente» (ibid.: 30).

Por lo tanto, la labor de un docente de lengua y de E/LE no pasa solo por enseñar contenidos específicamente lingüísticos, como los gramaticales, sino también por tratar de mejorar sus habilidades comunicativas y los procesos de pensamiento que se emplean para un fin concreto (Durán et al., 2009), más aún si parte de nuestro alumnado no es nativo en español. De esta forma, cualquier aprendizaje debe ser concebido con un sentido, es decir, dando las suficientes coordenadas contextuales para que los estudiantes comprendan la finalidad del tipo de texto que se les pide (Hymes, 1989).

Estas directrices deben plantearse desde el enfoque comunicativo, un modelo que no nació con la finalidad de relegar a los métodos tradicionales, sino que «readapta principios pedagógicos de métodos o aproximaciones metodológicas previas [...] optimizados de manera ecléctica» (Maati, 2013: 114). En él, se da más importancia a las capacidades de expresión y comprensión tanto oral como escrita, desarrolladas a partir del estudio y creación de textos auténticos y reales (Maati, 2013; Li, 2016).

Por su parte, con el fin de que se consiga la asimilación de un tipo de género discursivo por parte de los estudiantes, se deben enseñar los pasos necesarios que consigan su completo desarrollo, dando lugar, por un lado, a tareas posibilitadoras que aborden un aspecto del producto que se espera conseguir y, por otro, a tareas comunicativas que pretenden poner en funcionamiento todo aquello que el estudiante ha aprendido.

Esta división de actividades se centra en el llamado enfoque por tareas (Doughty, 2000), que se propone parcelar el proceso de producción en tareas comunicativas que 
involucren a los estudiantes en la preparación y producción de textos atendiendo, fundamentalmente, a su sentido más que a su forma (Nunan, 1989). En esta misma línea, Estaire y Zanón (1990) sostienen que estas tareas deben cumplir una serie de requisitos para considerarse comunicativas: 1) ser fieles a los procesos de comunicación de la vida real, 2) que se constituyan como actividades unitarias y 3) que tengan un objetivo claro sobre el aprendizaje de la lengua.

Sobre estas bases se construyó la secuencia base destinada al conocimiento de un género discursivo oral (el anuncio). No obstante, fue evidente que no obtuvo el resultado más deseable en todo el conjunto de los alumnos, hecho que ha llevado a repensar la actuación dada. Para ello, se tratará de visualizar los problemas del alumnado y buscar su correspondencia con las dificultades que puedan plantear los materiales.

\subsection{EI análisis de errores y de actuación}

La realidad de las aulas impone siempre un constante ensayo y error en el que el profesorado trata de encontrar la metodología más adecuada para que sus estudiantes alcancen las metas establecidas. Por esta razón, se hace necesario el proceso de reflexión y análisis de los errores cometidos por nuestros estudiantes y, especialmente, de los que hemos cometido nosotros en el diseño e implementación de los materiales y la metodología de aula.

De esta forma, se sigue un proceso de retroalimentación en el que el error se erige como objeto de estudio y no como enemigo del aprendizaje, y por esta razón:

son ahora las manifestaciones concretas de los aprendices las que centran el interés de los estudios; el cambio metodológico es crucial: se pasa de las predicciones desde el plano de la abstracción —en el que hasta ahora se habían desarrollado las investigaciones en L2 - al espacio real y concreto de las producciones de los discentes, con el objeto de obtener datos empíricos que favorezcan la explicación de los errores en el proceso de adquisición y aprendizaje de las lenguas extranjeras. (de Alba, 2009: 3)

Como se ha mencionado, otro aspecto relevante en el proceso de reflexión es el análisis de la actuación del profesorado y de los materiales diseñados, ya que se debe observar en qué medida contribuyen al desarrollo del conocimiento de nuestros alumnos (Romero y Gómez, 2015). Por tanto, un análisis efectivo tomará en consideración los errores del alumnado como punto de partida para cuestionarse qué ha fallado en la implementación de la secuencia para, posteriormente, proponer las mejoras respectivas.

\section{Diseño de la secuencia}

El planteamiento que se siguió parte tanto del enfoque comunicativo (Canale-Merril, 1996; Maati, 2013) como del enfoque por tareas (Nunan, 1989; Abelló, 2010), en los que se defiende que para lograr la plena adquisición del género discursivo debe, no solo explicarse, sino también practicarse. Esta práctica debe llevarse a cabo ofreciendo los ingredientes necesarios para lograr que, de manera autónoma, los estudiantes sean capaces de producir el texto que se ha desarrollado durante la secuencia (Nunan, 1989).

A partir de estas bases, autores como Doughty (2000) señalan que el proceso de creación de un texto debe tener tres fases: conocimiento del género, preparación del 
texto (realización del guion y revisión) y difusión. Dentro de la secuencia didáctica que se diseñó ${ }^{2}$, se intentó seguir el mismo planteamiento (ver imagen 1): la primera parte se destinó a introducir a los alumnos al género del anuncio publicitario y a las estrategias mayormente empleadas en este tipo de discurso. La segunda parte se centró en tratar de crear un guion para un spot publicitario. En él, los alumnos tuvieron que centrar el tema sobre el que versaba su anuncio y se redactó un primer texto inicial. Luego, se trataron una serie de actividades centradas en el conocimiento del género y el desarrollo de las herramientas necesarias para redactar el suyo propio, así como la revisión de los textos que se iban construyendo, entre las que se incluía una actividad para reconocer tipos de argumentos en canciones de malvados de películas y la visualización y debate del anuncio Amodio.

Cabe decir que estas actividades se concibieron de manera colaborativa, es decir, cada texto fue construido por un grupo de unos 5 estudiantes, en el que todos tuvieron que colaborar en las distintas fases de creación del anuncio (búsqueda de información, redacción del borrador, corrección...). Además, todos los alumnos contaban con un diario de sesiones en el que debían reflejar los contenidos aprendidos y los aspectos que mejorarían de las clases.

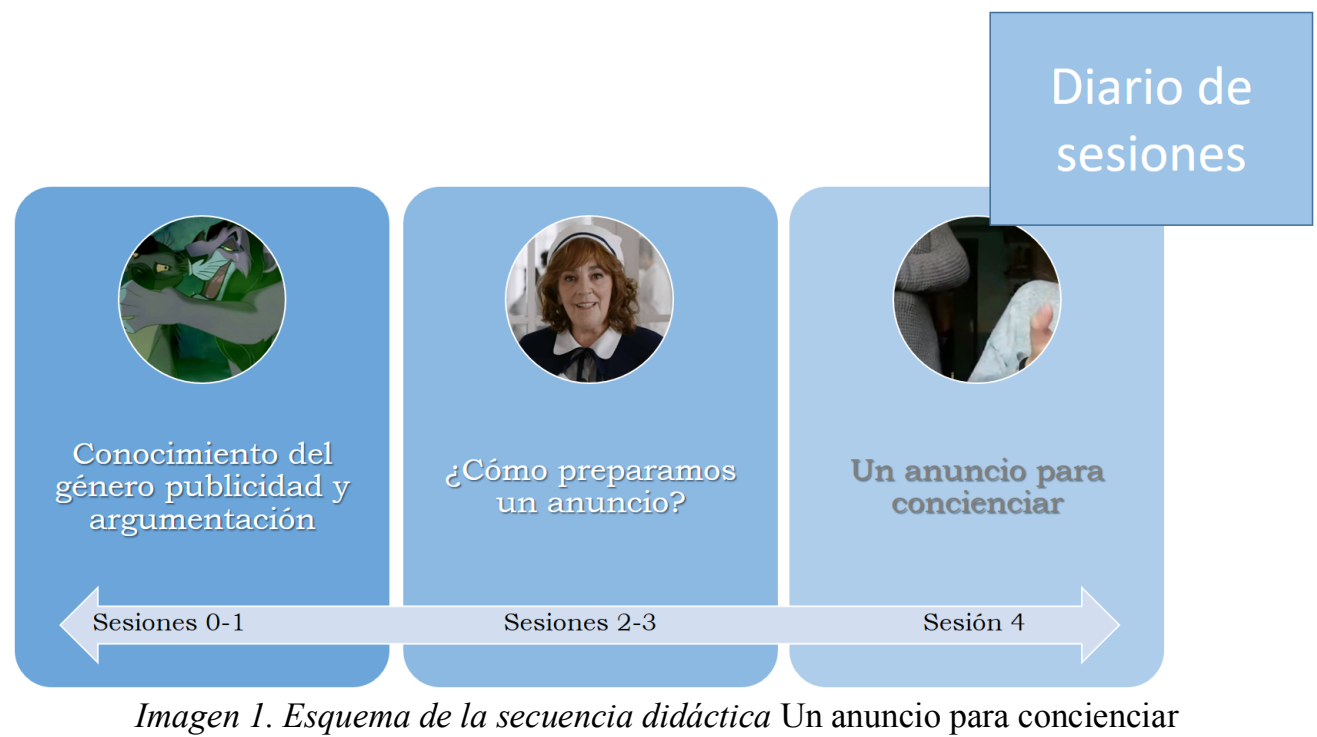

El objetivo final, por tanto, versaba en la creación de un anuncio publicitario que podía realizarse de dos formas:

Opción A: a través de un videomontaje.

Opción B: a través de una representación en el aula.

Para lograrlo, se pretendió que los estudiantes conociesen ciertos tipos de argumentación empleados usualmente en la publicidad, las habilidades necesarias para preparar una intervención oral adecuada y los recursos lingüísticos necesarios que estén vinculados tanto con el género como con estrategias generales de expresión oral.

A partir de los resultados recopilados a través de la secuencia, en el siguiente apartado se mostrará el análisis realizado de los errores más frecuentes cometidos por los

\footnotetext{
${ }^{2}$ Pueden consultarse los materiales de la secuencia en: https://drive.google.com/drive/folders/16WdL07dRpsejaOzP4fRdAiNTTOokZMPh?usp=sharing
} 
alumnos no nativos de clase y las dificultades que pudiesen plantear los materiales diseñados. Para ello, se darán una serie de trazas del contexto en el que se implementó la secuencia y se mostrarán distintos ejemplos en los que los materiales eran ineficaces.

\section{4. ¿Qué ha fallado? Análisis de los errores cometidos durante la secuencia}

Las actuaciones reales siempre ponen a prueba las utopías diseñadas, esta vez en forma de secuencia didáctica. El choque con la realidad y los distintos contextos nos obligan a enfrentarnos a las fallas de nuestros materiales. Esta secuencia se desarrolló en dos grupos de tercero de la ESO que estaban conformados por 18 y 22 estudiantes, respectivamente. El primero de ellos solo contaba con un alumno no nativo, mientras que el segundo contaba con 7 alumnos que estaban aprendiendo aún el español, razón por la que resulta más interesante analizar este grupo. Por su parte, el nivel de adquisición de ese alumnado era variable en ambos grupos, yendo desde un A2 hasta un B2 entre ambos grupos.

Los apartados que se van a tener en cuenta para el análisis se distribuyen en algunas tareas que los estudiantes tuvieron que realizar a lo largo de la secuencia. Se empezará con la tarea final, el anuncio, que constituye el material más evidente para comprobar el nivel de adquisición del alumnado. Posteriormente, en base al producto final se podrán analizar el resto de materiales que han llevado a este, siguiendo una distribución inductiva que lleve a buscar la raíz de los problemas encontrados en cada grupo. Cabe señalar, por último, que los materiales que se ofrecen han sido anonimizados y que las imágenes se han emborronado previamente para preservar el anonimato de los estudiantes ${ }^{3}$.

\section{a) La tarea final: un anuncio para concienciar}

El primer punto de análisis fue observar las evaluaciones que recibieron los alumnos de su proyecto final (un anuncio), que se hicieron a través de una rúbrica de la que eran partícipes tanto la profesora como los estudiantes. Esta rúbrica estaba dividida en 4 secciones: 1) Expresión Oral, 2) Expresión corporal, 3) Adecuación al género anuncio, 4) Argumentación del anuncio. Cada una de ellas tenía una serie de ítems con distintas opciones según el grado de adquisición del grupo en cuestión. No obstante, para este artículo nos interesa especialmente la evaluación de los alumnos no nativos. Por esta razón, aunque se muestren los resultados de todos los estudiantes, se hará hincapié en las dificultades de los aprendices de español.

\begin{tabular}{|l|l|l|l|l|l|}
\hline Alumnos & $\begin{array}{l}\text { Expresión } \\
\text { oral (4) }\end{array}$ & $\begin{array}{l}\text { Adecuación al } \\
\text { género (3) }\end{array}$ & $\begin{array}{l}\text { Argumentación } \\
(\mathbf{2})\end{array}$ & $\begin{array}{l}\text { Expresión } \\
\text { corporal (1) }\end{array}$ & Total \\
\hline Nativos & 3.25 & 1.31 & 1.44 & 0.56 & 6.37 \\
\hline No nativos & 2.05 & 1.08 & 1.37 & 0.49 & $4.99(5) / 10$ \\
\hline
\end{tabular}

Tabla 2. Nota media de todos los estudiantes

Como se puede comprobar a través de la tabla, las diferentes notas conseguidas por los estudiantes residen, principalmente, en la expresión oral y en la adecuación al género

\footnotetext{
${ }^{3}$ Para más información: links.uv.es/lopd/derechos
} 
discursivo anuncio; ya que en la expresión corporal y en la argumentación no se aprecian diferencias significativas en el apartado numérico de la evaluación.

No obstante, centrando la mirada en los anuncios, se puede comprobar que la expresión corporal del alumnado no nativo se vincula a una falta de seguridad en el idioma y en el contenido que se estaba tratando, ya que su gesticulación oscilaba entre el nerviosismo y lo estático, además de buscar apoyos para su interpretación ${ }^{4}$. Como puede observarse en la siguiente imagen:

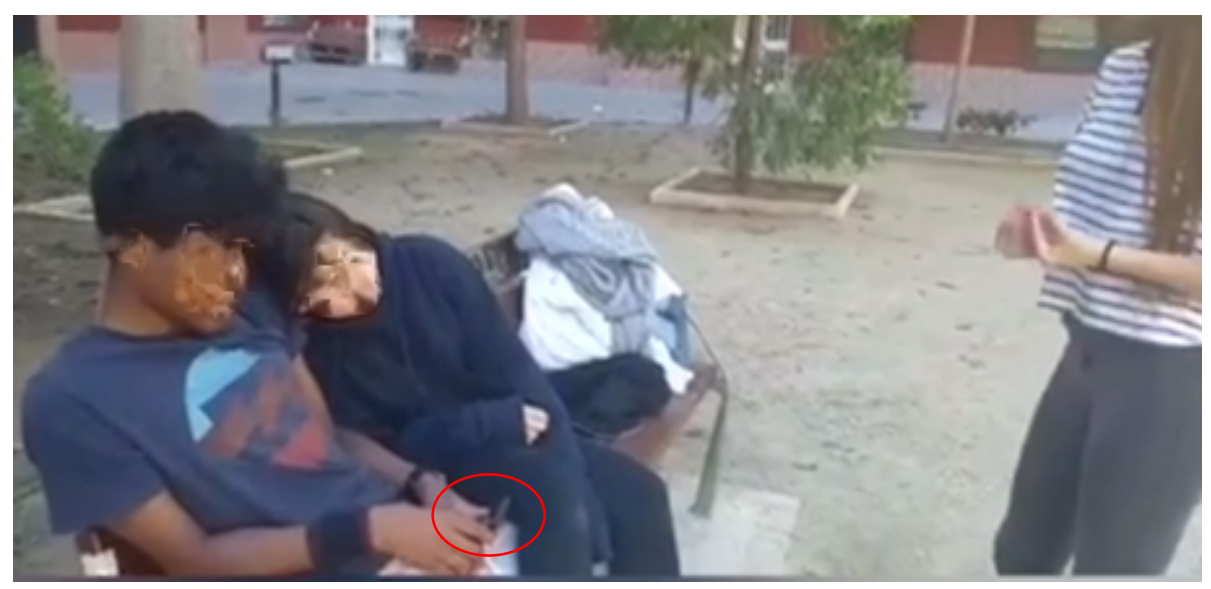

Imagen 2. Imagen anuncio grupo 2, clase B

(1) A: no / pero esquee / estaba dando una vuelta por el parque yy os he visto tan juntito y no sé me ha parecido muy extraño quee / dos chicoss / pues estén juntos y tú encima / ((eres)) tan diferente a nosotros / ¿sabes?

B: estoy grabando toda esta conversación para ((ir)) llamar a la policía

La escena trataba de representar a una pareja del mismo sexo y de distinta etnia sentados en el parque que es interrumpida por la presencia de una persona que les increpa por lo extraño de su relación. En esa circunstancia, uno de los novios informa a la otra persona que está siendo grabada para ser denunciada (B). Esta acción se emplea de excusa para que el alumno con dificultades pueda tener el móvil en las manos, del cual no aparta la vista en ningún momento (probablemente, porque tenía su línea de texto escrita en él por si se le olvidaba). Una situación similar, aunque más evidente, se puede observar en el siguiente ejemplo:

\footnotetext{
${ }^{4}$ Con apoyos nos referimos a cualquier medio que les permitiera tener el texto del guion del anuncio que estaban grabando. En concreto, en la imagen 2 se observa al estudiante sosteniendo y mirando su móvil.
} 


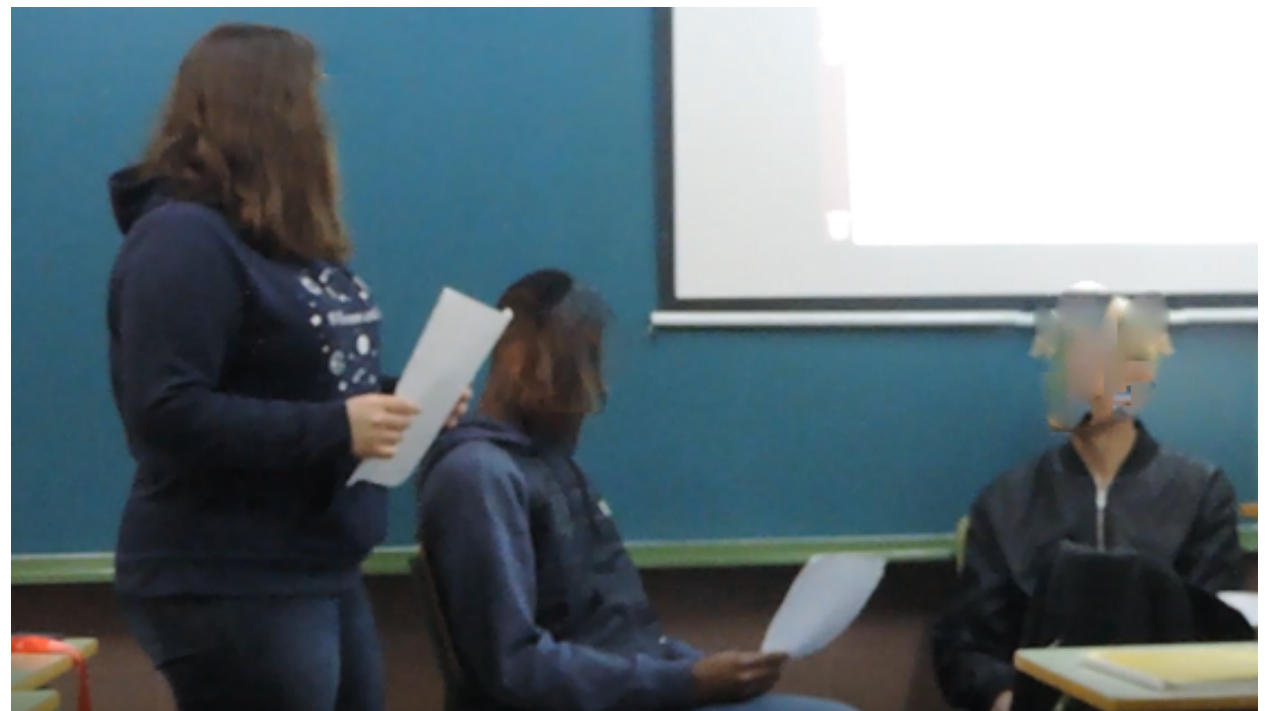

Imagen 3. Anuncio grupo VI, clase 2

En esta ocasión, los alumnos decidieron representar el anuncio en directo delante de sus compañeros de clase. Dejando a un lado la ausencia de cualquier tipo de recurso escénico y de cualquier organización de los actores, se puede observar que ha habido una falta de preparación que se suma, además, a su falta de competencia en la lengua de la asignatura. Su escena pretendía recrear una conversación en un parque entre tres personas que debatían sobre la calidad del aire para exponer un par de datos sobre la contaminación. Sin embargo, su anuncio pierde fuerza porque dos de los integrantes, curiosamente los dos no nativos, se aferran al papel y no actúan prácticamente.

Como se puede comprobar, el principal problema para el alumnado no nativo reside ya no en el nivel de conocimientos o de dominio del idioma, sino en la ausencia de seguridad en sus discursos que les lleva a depender de materiales de apoyo constantemente. Cabe examinar, asimismo, cómo fue el seguimiento de estos a través del diario de sesiones que puede arrojar nuevas pistas sobre los problemas que experimentaron estos estudiantes.

\section{b) Diario de sesiones}

Como se ha mencionado, durante la secuencia se entregó una ficha/diario de seguimiento de las sesiones en los que cada estudiante debía reflejar qué había aprendido en la clase y qué elementos consideraban más difíciles desde su punto de vista. Sin embargo, parte de los estudiantes, especialmente los no nativos, encontraron dificultades para expresar sus pensamientos y, posiblemente, para comprender qué debían escribir en él. Si se observan los siguientes ejemplos: 


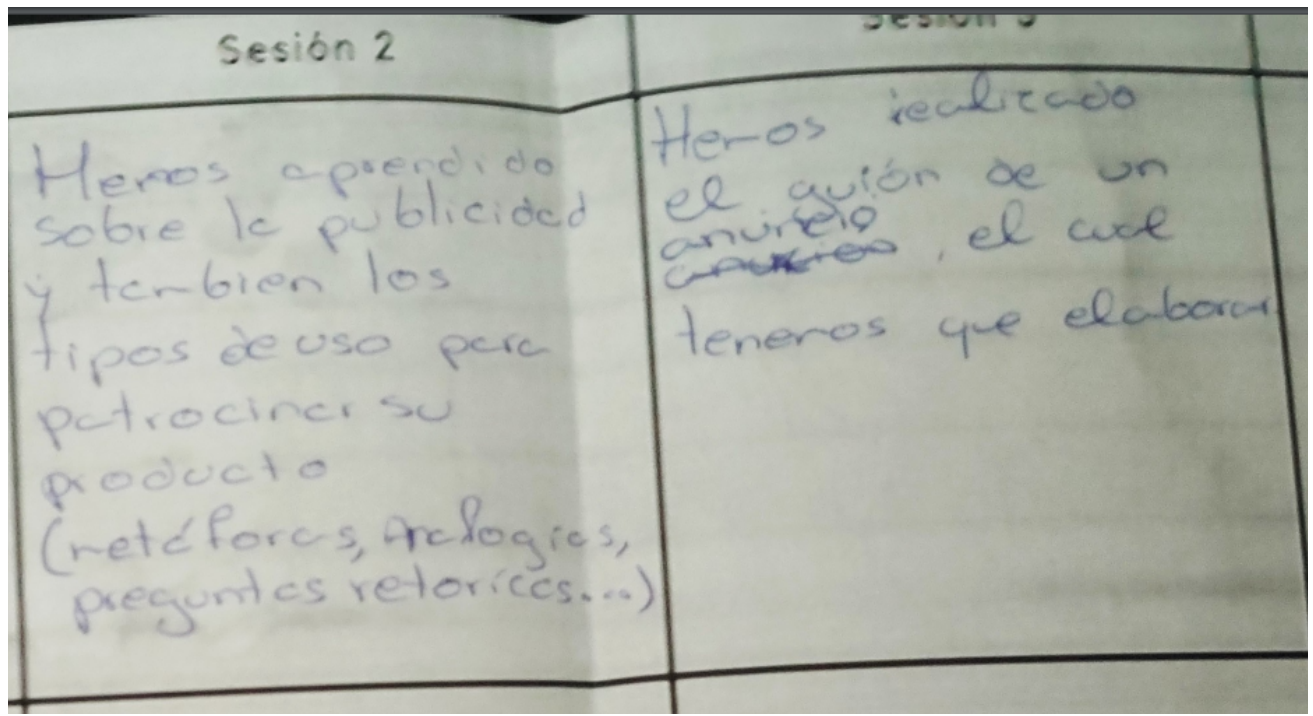

Imagen 4. Diario de sesiones (I) - nativo

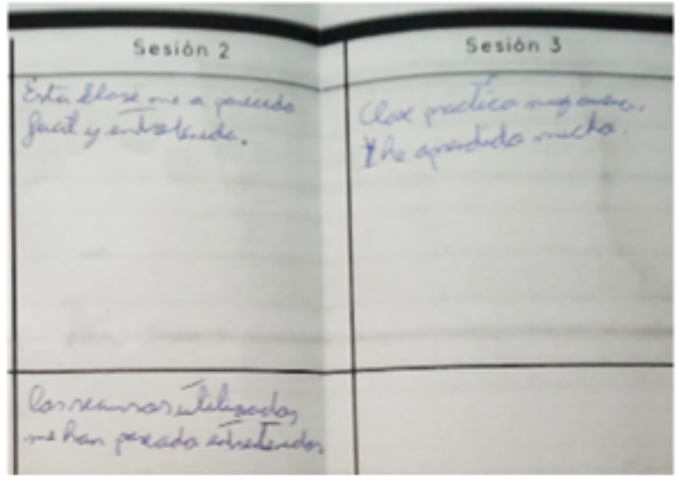

Transcripción literal

\begin{tabular}{|l|l|}
\hline \multicolumn{1}{|c|}{ Sesión 2 } & \multicolumn{1}{c|}{ Sesión 3 } \\
\hline $\begin{array}{l}\text { Esta clase me a } \\
\text { parecido facil y } \\
\text { entretenida }\end{array}$ & $\begin{array}{l}\text { Clase practica muy } \\
\text { amena, he } \\
\text { aprendido mucho }\end{array}$ \\
\hline $\begin{array}{l}\text { Los recursos } \\
\text { utilizados me han } \\
\text { parecido } \\
\text { entretenidos }\end{array}$ & \\
\hline
\end{tabular}

Imagen 5. Diario de sesiones (II) - no nativo

Los dos ejemplos nos muestran parte del diario de sesiones de un alumno nativo y otro de uno no nativo. En esta ocasión, el primero ha tratado de hacer un breve resumen de las clases y de los elementos que ha aprendido (aunque mal clasificados, ya que denomina «usos» a los «recursos»), mientras que el segundo solo ha reflejado un sentimiento sobre la clase y no ha reseñado ni lo que ha aprendido ni las dificultades que le han presentado. Si tenemos en cuenta el siguiente ejemplo:

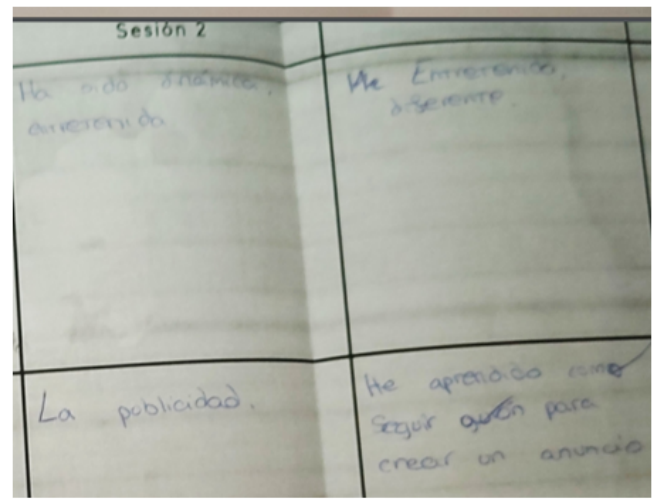

Transcripción Literal:

\begin{tabular}{|c|c|}
\hline Sesión 2 & Sesión 3 \\
\hline $\begin{array}{c}\text { Ha sido dinámica, } \\
\text { entretenida }\end{array}$ & $\begin{array}{c}\text { Me entrenenido, } \\
\text { diferente }\end{array}$ \\
\hline La publicidad & $\begin{array}{c}\text { He aprendido come } \\
\text { seguir gution para crear } \\
\text { un anuncio }\end{array}$ \\
\hline
\end{tabular}

Imagen 6. Diario de sesiones (III) - no nativo 
Este estudiante sigue escribiendo sus sentimientos con respecto a la clase («entretenida» $\mathrm{y}$ «diferente»), pero ha tratado de reflejar qué se estaba trabajando, con mayor o menor acierto. Sin embargo, se impone la sencillez y no ahonda en qué contenidos se han aprendido específicamente durante la sesión.

Esta clase de errores son síntoma de varios problemas: por un lado, parece que los estudiantes no han comprendido qué se debe hacer con el diario y han tratado de expresar lo que, en su forma personal, se entiende por uno de ellos (un lugar donde reflejar nuestros sentimientos y percepciones). Por otro, el alumnado no nativo que sí ha entendido qué se debe hacer, parece que ha comprendido solo las líneas más generales de la clase (se estaba hablando, efectivamente, de publicidad), pero no ha asimilado los contenidos concretos (los recursos que se emplean en los anuncios).

c) Ficha de debate

Por último, se centrará la mirada en la ficha de debate que se empleó para analizar un anuncio con doble temática social-comercial (Amodio). Los alumnos tuvieron que responder a una serie de preguntas en grupo que, posteriormente, se pusieron en común con toda la clase. No obstante, de nuevo, se detectan algunos problemas con el alumnado no nativo:

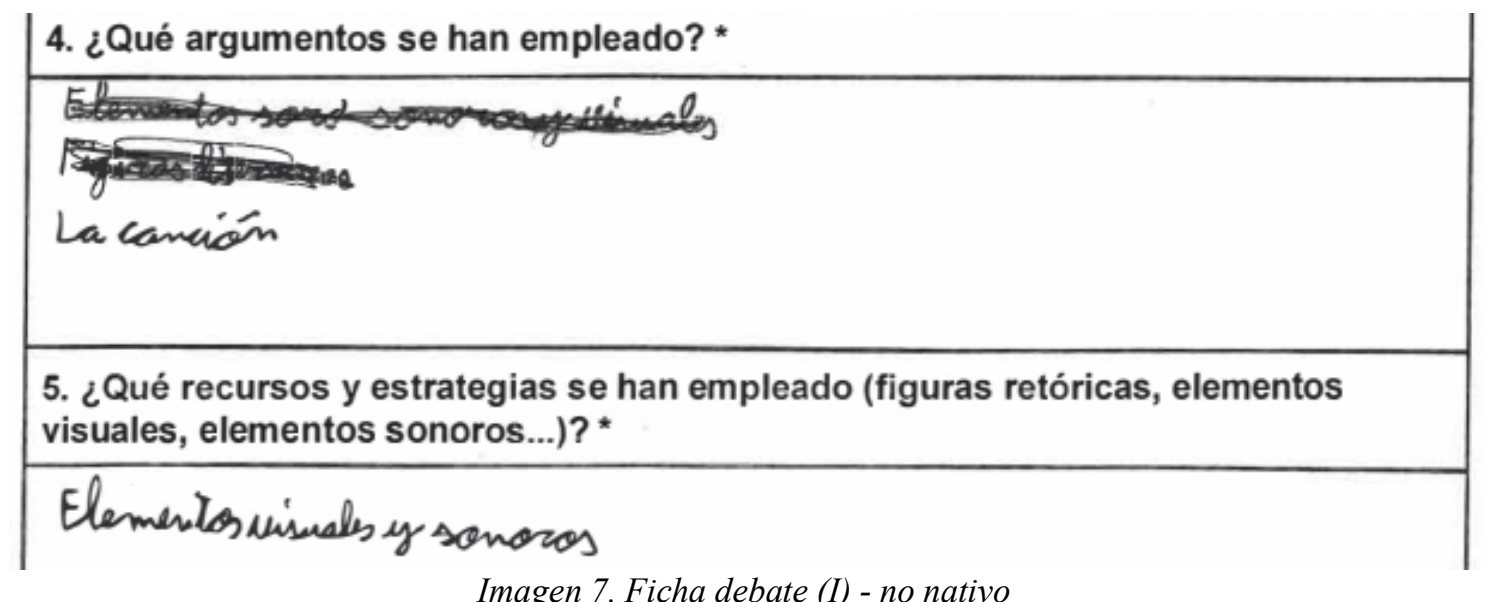

Imagen 7. Ficha debate (I) - no nativo

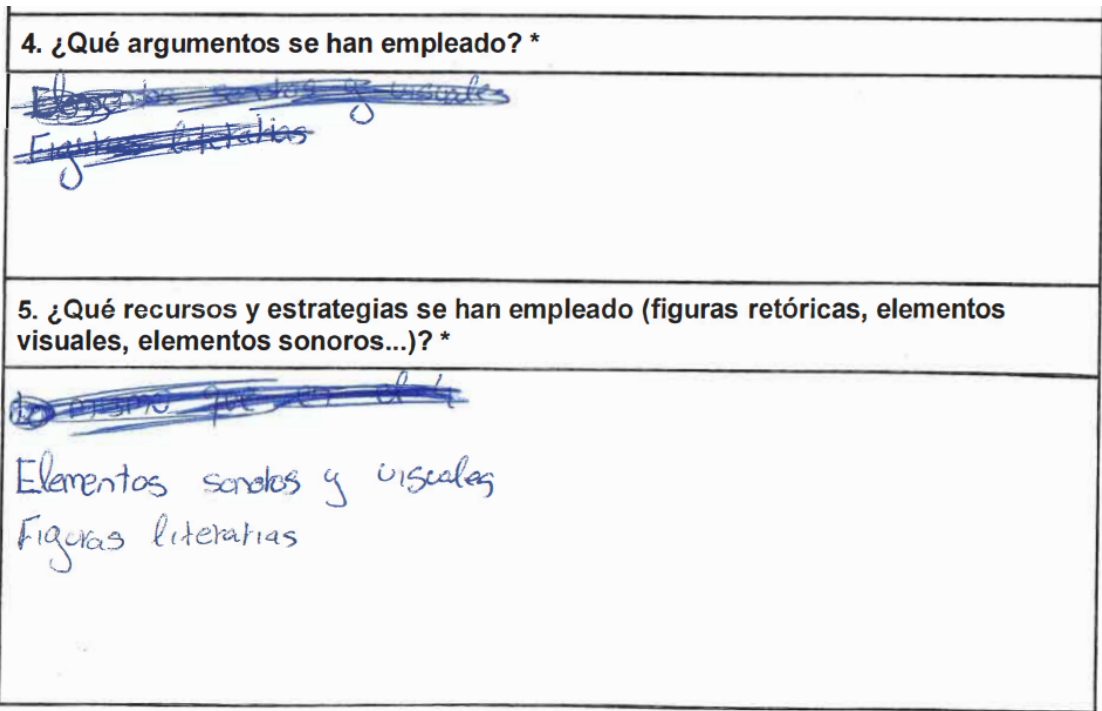

Imagen 8. Ficha de debate (II) - no nativo 


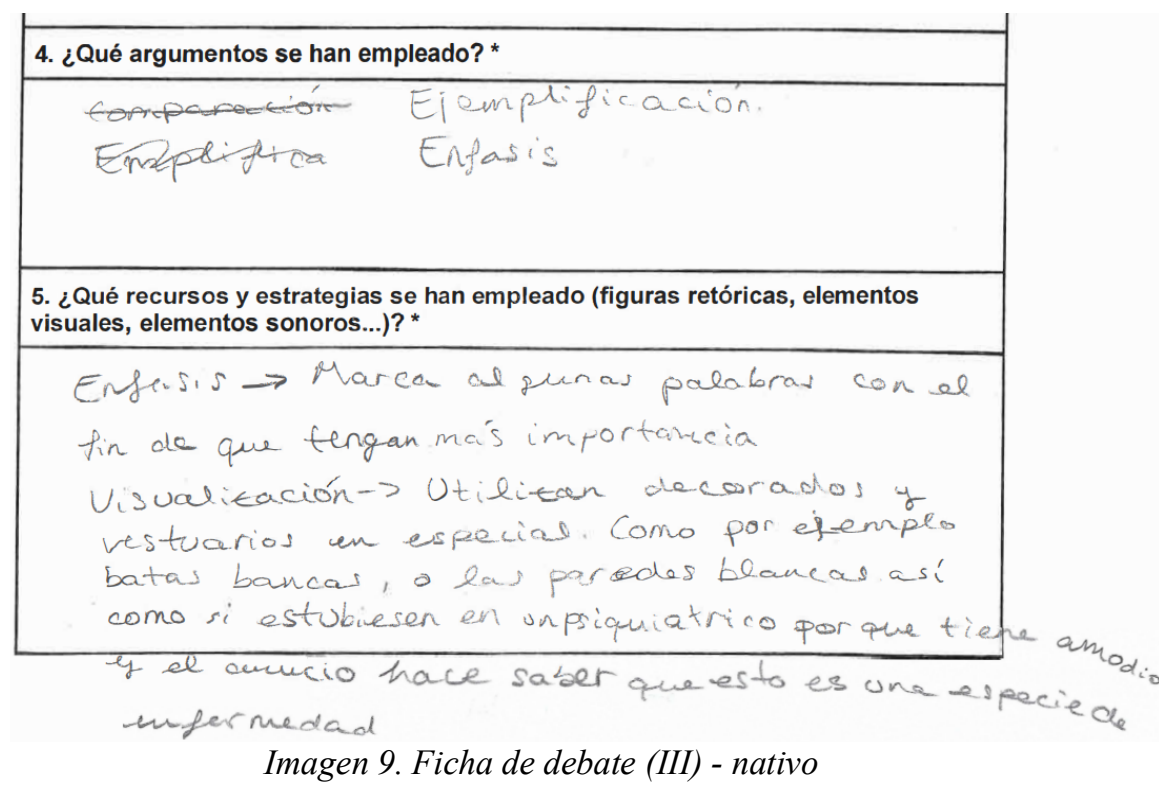

Como se puede observar en los dos ejemplos de los alumnos no nativos ( 7 y 8 ), se han limitado casi exclusivamente a repetir las palabras que había en los enunciados sin aportar ninguna explicación más detallada ni elaborar frases más complejas (por ejemplo, relatando el momento en el que se usan, como ha realizado el alumno nativo en la pregunta 5 [imagen 9]). Todo parece apuntar a una falta de competencia en el ámbito escrito que se traduce, de nuevo, en un déficit de seguridad que les lleva a actuaciones incorrectas o poco precisas.

Atendiendo a todos los errores que se han ido observando, es posible determinar que el alumnado se vea altamente influenciado por su inseguridad, más allá de la competencia que puedan tener en la lengua vehicular de la clase. A partir de estas consideraciones, se ha realizado una propuesta de mejora que pretende focalizar la atención en estos aspectos problemáticos y acercarlos a todo el alumnado del aula (nativo o no).

\section{Propuesta de mejora de la secuencia ${ }^{5}$}

Los resultados del análisis anterior proporcionan una base sobre la que proponer una serie de mejoras que traten de paliar las dificultades que el alumnado no nativo ha experimentado a lo largo de esta secuencia didáctica. Los aspectos que deben modificarse se dividen en tres grupos:

\section{a) Temática}

En primer lugar, si bien es cierto que la temática empleada (causas sociales) podía ser más o menos cercana a todos los estudiantes y que permitía desarrollar la conciencia social del alumnado (objetivo fundamental al que todo profesor debe aspirar, según el currículum); generaba cierta preocupación en todos los grupos, ya que se sintieron inseguros en el momento de escoger su causa central y, posteriormente, en los recursos

5 Pueden consultarse los nuevos materiales en: https://drive.google.com/drive/folders/1biz1DdFtNQcrbJw6SGk5NQDUUHucGC4?usp=sharing 
que debían emplear para sustentar su anuncio. Este problema general pudo achacarse aún más en el alumnado no nativo.

De esta manera, se plantea la posibilidad de modificar la temática del anuncio, de tal forma que sea motivador para todos los estudiantes y que sea más cercano a ellos (Cea Álvarez, 2018: 51). Por ello, se modifica este y se propone que los estudiantes realicen un anuncio sobre destinos turísticos, haciendo especial hincapié en los aspectos culturales y sociales del sitio que se pretende anunciar.

Sin embargo, se añade una restricción: los estudiantes nativos tendrán que escoger destinos relacionados con los países de origen de los alumnos inmigrantes y viceversa, los estudiantes no nativos deberán escoger algún lugar perteneciente a la geografía española. Con ello, todos los grupos de trabajo (ver sección c) serán no expertos en el tema de su anuncio, pero expertos en algún tema de los otros grupos. Además, dando pie a esta nueva dinámica se consigue que todos los integrantes se adentren y conozcan un poco más las culturas vecinas.

\section{b) Recursos y materiales}

Otro aspecto que puede modificarse atañe al diseño de los materiales y recursos que se han empleado a lo largo de la secuencia. Estas modificaciones deben atender a tres criterios que se vinculan con lo visto en el análisis de errores: 1) facilitar la comprensión de los enunciados, 2) dar suficiente material de ejemplo para facilitar la construcción de enunciados complejos y 3 ) ofrecer los contenidos de la secuencia en múltiples formatos que los conviertan en más accesibles.

En primer lugar, se debe facilitar una comprensión suficiente de los enunciados de las actividades que se proponga a los estudiantes sin añadir elementos que sean superfluos o que puedan llevarlos a la confusión. Por esta razón, se propone una modificación de la actividad de debate en la que se cambien dos partes: por un lado, con el fin de adecuar el anuncio a la nueva temática de la secuencia, Amodio pasará a ser la Campaña de Turismo de Madrid 2013, en el que se promociona la cultura y se reseñan los aspectos más relevantes de la ciudad. Por otro, se modificarán ciertos enunciados de la ficha de análisis para el debate respetando la máxima de claridad, sencillez e informatividad; aunque evitando que el contenido sea demasiado general y vago:

\begin{tabular}{|l|l|}
\hline Enunciado Anterior & Nuevo Enunciado \\
\hline $\begin{array}{l}\text { 4. ¿Qué argumentos se han } \\
\text { empleado? }\end{array}$ & $\begin{array}{l}\text { 4. ¿Qué argumentos se han empleado para convencer al } \\
\text { público de que viajen a Madrid? }\end{array}$ \\
\hline $\begin{array}{l}\text { 5. ¿Qué recursos y estrategias se han } \\
\text { empleado (figuras retóricas, } \\
\text { elementos visuales, elementos } \\
\text { sonoros,...)? }\end{array}$ & $\begin{array}{l}\text { 5. En el anuncio, vemos que se muestran imágenes de } \\
\text { los lugares importantes de Madrid, iqué recursos y } \\
\text { estrategias se han empleado para volver atractiva esta } \\
\text { ciudad? } \\
\text { Ejemplo: se emplea una canción con un ritmo alegre y } \\
\text { que invita a la diversión. }\end{array}$ \\
\hline
\end{tabular}

Tabla 3. Ejemplo de modificación de preguntas en la ficha de debate

Asimismo, en la evaluación final, todos los grupos van a ser expertos y no expertos en los apartados correspondientes a la temática, el único que se ha añadido a la rúbrica 
creada para la secuencia base. En cuanto a los apartados referentes a las competencias comunicativas y de adquisición del género discursivo, se proponen leves modificaciones que atañen, principalmente, a facilitar la comprensión de los enunciados para todos los estudiantes, tengan el nivel que tengan de español.

\section{Temática}

¿El anuncio muestra partes importantes de vuestro país o de vuestra ciudad?

a. Sí, ha mostrado las partes más importantes que les recomendamos (incluso se han informado de más).

b. Algunas partes eran importantes, aunque otras eran menos relevantes.

c. No han mostrado nada interesante de nuestro país.

¿Se han mostrado y contado los detalles culturales más interesantes?

a. Sí, han mostrado una parte de nuestra cultura, aunque sea pequeña; pero bien documentada.

b. Han mostrado parte de nuestra cultura, pero no se han informado o seguido nuestras recomendaciones

c. No han mostrado nada que tenga que ver con nuestra cultura

Tabla 4. Nuevo apartado de la rúbrica

\begin{tabular}{|c|c|}
\hline \multicolumn{2}{|c|}{ Expresión oral } \\
\hline Enunciado anterior & Nuevo enunciado \\
\hline $\begin{array}{l}\text { Vocabulario empleado: } \\
\text { a. Se emplea un vocabulario claro y variado, adecuado al } \\
\text { tema que se trata. } \\
\text { b. Se emplea un vocabulario claro y variado, con alguna } \\
\text { repetición, adecuado al tema que se trata. } \\
\text { c. Se emplea un vocabulario poco específico y repetitivo, } \\
\text { adecuado al tema que se trata. } \\
\text { d. Se emplea un vocabulario no adecuado al tema que se } \\
\text { trata. }\end{array}$ & $\begin{array}{l}\text { Vocabulario relacionado con el tema del } \\
\text { anuncio (viajar, cultura, arte...): } \\
\text { a. Se emplea un vocabulario claro y variado, } \\
\text { adecuado al tema que se trata. } \\
\text { b. Se emplea un vocabulario claro y variado, con } \\
\text { alguna repetición, adecuado al tema que se trata. } \\
\text { c. Se emplea un vocabulario poco específico y } \\
\text { repetitivo, adecuado al tema que se trata. } \\
\text { d. Se emplea un vocabulario no adecuado al tema que } \\
\text { se trata. }\end{array}$ \\
\hline $\begin{array}{l}\text { Adecuación al registro: } \\
\text { a. Se utiliza un lenguaje formal, adecuado al tema. } \\
\text { b. Se emplea un lenguaje formal y adecuado al tema, } \\
\text { aunque con ciertos coloquialismos. } \\
\text { c. Se emplea un lenguaje que se adecua al tema, aunque no } \\
\text { formal. } \\
\text { d. Se emplea un lenguaje coloquial sin ninguna vinculación } \\
\text { al tema. }\end{array}$ & $\begin{array}{l}\text { Adecuación al registro (una forma de hablar } \\
\text { cercana pero evitando errores y } \\
\text { vulgarismos): } \\
\text { a. Se utiliza un lenguaje formal, adecuado al tema. } \\
\text { b. Se emplea un lenguaje formal y adecuado al tema, } \\
\text { aunque con ciertos vulgarismos. } \\
\text { c. Se emplea un lenguaje que se adecua al tema, } \\
\text { aunque no formal. } \\
\text { d. Se emplea un lenguaje coloquial sin ninguna } \\
\text { vinculación con el tema. }\end{array}$ \\
\hline
\end{tabular}

Tabla 5. Ejemplo de modificación de la rúbrica

\section{c) Organización de la secuencia y distribución de los grupos}

Por último, cabe reseñar que, siguiendo la premisa de que todo el alumnado debe ser no experto en el tema escogido para su tarea final, debe de distribuirse a estos de forma en la que los estudiantes no nativos se concentren en grupos separados de los nativos. Esta medida que puede parecer contraproducente a priori permitirá configurar, en primer lugar, una atención individualizada a cada tipo de grupo $\mathrm{y}$, posteriormente, crear dinámicas de cooperación entre las distintas agrupaciones. 
La metodología de implementación que se seguirá, por tanto, partirá de que los grupos no nativos deberán hacer un anuncio sobre algún territorio español, mientras que el resto deberán hacerlo sobre algún país de origen de los alumnos inmigrantes. Por lo tanto, la fase de corrección de guiones de los anuncios pasará a convertirse en una o dos sesiones de asesoramiento entre los grupos para recolectar información y contrastar los guiones que se van creando.

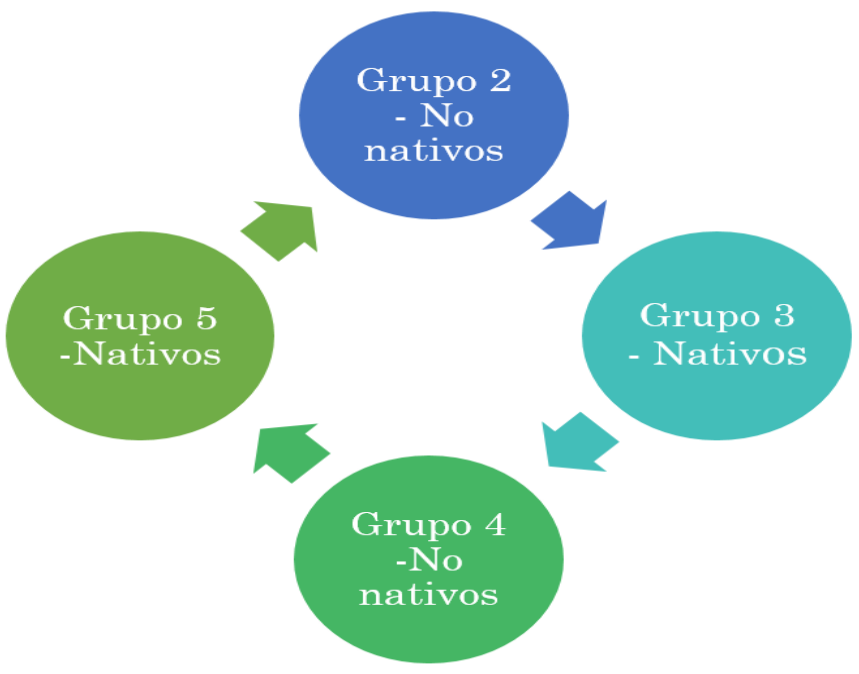

Esquema 1. Organización de los grupos de trabajo

Como se había mencionado en la sección anterior, este hecho permitirá que todos los grupos se sientan expertos en, al menos, uno de los anuncios que sus compañeros van a presentar; hecho que les dará una mayor seguridad y confianza en el momento de utilizar la rúbrica con sus compañeros. Por ello, aunque la evaluación sea conjunta, el grupo experto en el tema del anuncio que se exhiba será el encargado de centrar la mirada en profundidad en cómo se ha mostrado la ciudad/país en la tarea.

Por otra parte, el desarrollo de la secuencia contemplaba que al finalizar cada clase los estudiantes expresaran en su ficha/diario los contenidos que habían aprendido durante el día y/o las propuestas de mejora que ellos consideraran. Sin embargo, el alumnado con dificultades en español solo se limitó a expresar sus sentimientos (vocabulario, normalmente, cercano y que se aprende primero según el Plan Curricular del Instituto Cervantes [2006]). Por esta razón, se propone dedicar unos 10 minutos al inicio de cada sesión para comentar aquello que se ha escrito en los diarios $\mathrm{y}$, de esta forma, retroalimentar a los estudiantes con mayores dificultades para seguir los contenidos y, en consecuencia, reflejarlos en sus respectivos diarios.

En resumen, las modificaciones que se han propuesto en los tres apartados han tratado de corregir ciertos aspectos de la secuencia (la temática, el diseño de algunos materiales y la organización de la secuencia) con el fin de acercar los contenidos a todo el conjunto del alumnado. Si bien es cierto que se trata de una propuesta, no deja de estar fundamentada en la experiencia que se obtuvo al implementar su versión alpha en un aula real y, de ahí, todos los cambios aportados a lo largo de este artículo. 


\section{Conclusiones}

El artículo ha presentado la problemática de llevar a cabo una secuencia didáctica diseñada para secundaria en una clase donde existe alumnado no nativo de español. A pesar de los esfuerzos durante las clases para ayudar a todos los estudiantes, el análisis de errores ha demostrado que existen diferencias en la asimilación de los contenidos, sean curriculares o no, entre los alumnos que tienen como lengua materna el español (o los que llevan escolarizados en países hispanohablantes mucho tiempo) y entre los que no tienen un dominio de esta lengua. Estos errores se pueden clasificar en los siguientes tipos:

1) Falta de comprensión lectora de enunciados. Como se ha podido comprobar anteriormente, por ejemplo, en la imagen $7(\S 4 b)$ el alumnado no nativo no posee una competencia lectora completa en español, hecho que hace que sus contestaciones a ciertas cuestiones sean la repetición del enunciado que se le ha preguntado. $\mathrm{O}$, como se veía en la imagen $8(\S 4 b)$, donde el alumno en cuestión no lograba reflexionar acerca del tópico que se requería, sino que expresaba con el vocabulario que en mayor o menor medida dominaba, la respuesta a la pregunta.

2) Carencias en la competencia escrita. Este hecho se vincula con el punto anterior, puesto que no solo no entienden por completo lo que se les pregunta, dado que su dominio de la lengua no es suficiente para llevar a cabo la respuesta esperada; sino que, además, cometen errores gramaticales como se podía ver en la imagen 6: «me Ø entretenido*» $(\S 4 b)$.

3) Inseguridad lingüística. Los estudiantes no nativos, tengan un mayor o menor dominio de la lengua vehicular, sienten cierta ansiedad en las tareas, especialmente en aquellas que implican la oralidad. Este hecho se puede observar, especialmente, por los tipos de acciones y la gestualidad que transmiten en los videos como se ha visto en las imágenes 2 y 3 .

Más allá de los errores que puedan venir por las circunstancias personales de cada estudiante (independientemente de su lengua materna), la tarea de los profesores es adaptar y proponer mejoras de los materiales implementados (Roca Marín, 2002). Por esta razón, se han propuesto una serie de modificaciones de la secuencia base que permitan acercar a todo el conjunto del alumnado los contenidos curriculares que se están dando:

1) Se debe escoger un tema que permita convertir a todo el alumnado en experto y no experto al mismo tiempo, de tal forma que la cooperación entre ellos se haga necesaria.

2) Los materiales (como las fichas o la rúbrica de evaluación) deben ser accesibles para todo el alumnado, sin que ello comporte un perjuicio a los contenidos que se imparten.

Además, el diario de sesiones puede resultar beneficioso si se cambia la perspectiva desde la que se ha trabajado: no debería servir solo como alimentación del profesorado, sino que debería servir como retroalimentación para toda la clase $\mathrm{y}$, de esta forma, permitir a los alumnos con un menor nivel de español que se beneficien de las opiniones y comentarios del resto de sus compañeros. Cabe destacar como punto positivo la estructura y organización de los materiales de acuerdo con el enfoque por tareas y el 
enfoque comunicativo, ya que permitió, en cierta medida, que todo el alumnado se adapte y consiga realizar la tarea final de forma más o menos correcta.

Los análisis de errores de los estudiantes y de los profesores permiten, por tanto, ser conscientes de los procesos que han fallado o no durante el desarrollo de las clases, aunque es cierto que las secuencias didácticas que se creen e implementen no pueden esperar siempre a la práctica para comprobar cómo funcionan. Por esta razón, se debe procurar controlar el mayor número de variables posibles (especialmente referentes al tipo de alumnado y a sus necesidades) para evitar cometer esta clase de errores en el futuro.

Sin embargo, la realidad de las aulas dificulta tanto la atención individualizada como la grupal, ya que las circunstancias para dar clases son un poco más complejas que en un aula de enseñanza específica de idiomas, especialmente, el elevado número de estudiantes nos obliga, en muchas ocasiones, a considerarlos como un ente único y no permite atender a los diferentes niveles que se pueden llegar a agrupar en una única clase. Se suma, asimismo, la falta de materiales de ELE adaptados para el aula de secundaria que tengan en cuenta no solo al alumno nativo o no nativo por separado, sino que los integren y que ayuden a crear dinámicas de integración y cooperación entre ellos.

En definitiva, esta propuesta pretende aunar tres realidades presentes en todo tipo de aulas: 1) el planteamiento de una secuencia didáctica antes de su puesta en práctica (es decir, su conceptualización teórica), 2) su implementación y su aplicabilidad y 3) plantearse, tras llevarla al aula, si verdaderamente esa secuencia didáctica funciona como se había conceptualizado $\mathrm{y}$, en caso negativo, realizar un análisis de errores teniendo en cuenta, sobre todo, las posibles variables ocultas en las que no habíamos pensado. Dicho de otro modo, este artículo se dispone como un continuum de trabajo constante entre lo que se puede llegar a pensar, lo que se implementa y lo que realmente funciona: el error nunca es negativo, siempre da pautas de mejora.

\section{Bibliografía}

AlBA QuiÑones, A. de (2009). «El análisis de errores en el campo del español como lengua extranjera: algunas cuestiones metodológicas.» Revista Nebrija de Lingüística Aplicada, 5 (3), 1-16

ABelló i VIELlA, X. (2010). «L'avaluació de l'aprenentatge en un enfocament comunicatiu per tasques als nivells intermedi i de suficiencia», Llengua $i$ ús: revista tècnica de política lingüística, 47, pp. 52-59,

Cea Álvarez, A. M. (2018). "Cómo incluir la competencia estratégica en una programación de ELE para mejorar la expresión oral» en Cea Álvarez et al. (eds.) Investigación e Innovación en la Enseñanza de ELE, pp. 44-68.

CANAle, M., y Swain, M. (1980). «Theoretical bases of com-municative approaches to second language teaching and testing». Applied linguistics, 1 (1), pp. 1-47.

CANALE-MERrill, C. (1996). «Fundamentos teóricos de los enfoques comunicativos». Revista Signos-Teoría y Práctica de la educación.

Decreto 87/2015, de 5 de junio, del Consell, por el que establece el currículo y 
desarrolla la ordenación general de la Educación Secundaria Obligatoria y del Bachillerato en la Comunitat Valenciana. Diari Oficial de la Comunitat Valenciana, València, España, 10 de junio de 2015.

Doughty, C. y WiLliams, J. (1998). «Pedagogical coiches in focus on form», en C. Doughty y J. Williams (eds.), Focus on Form in Clasroom Second Language Acquisition (pp. 197-261). Cambridge: Cambridge University Press.

DurÁn, C., LóPez, I., SÁnchez-Enciso, J., y SEDILES, Y. (2009). La palabra compartida. La competencia comunicativa en el aula. Barcelona: Octaedro.

ESTAIRE, S., y ZANÓN, J. (1990). «El diseño de unidades didácticas en L2 mediante tareas: principios y desarrollo». Comunicación, lenguaje y educación, 2(7-8), 55-89.

GÓmEZ CAMACHO, A. (2014). «La enseñanza de la ortografía al alumnado inmigrante en Secundaria» en Bravo-García, Eva; Gallardo Saborido, Emilio J.; Santos de la Rosa, Inmaculada y Gutiérrez Rivero, Antonio (eds.) Investigaciones sobre la enseñanza del español y su cultura en contextos de inmigración, pp. 97-116. Universidad de Sevilla y Universidad de Helsinki: España.

Gutiérrez Cruz, A.; Agulló TomÁs, E.; Rodríguez SuÁRez, J. y Agulló ToMÁs, Ma S. (2004). «Discursos juveniles sobre inmigración: un análisis psicosociológico en estudiantes de ESO», Psicothema, v. 16, n. ${ }^{\circ}$ 3, pp. 384-390.

HyMES, D.H. (1972). «On Communicative Competence». En J.B. Pride y J. Holmes (eds.) Sociolinguistics. Selected Readings (pp. 269-293). Harmondsowrth: Penguin.

- (1989). «Ways of speaking». En R. Bauman y J. Sherzer (eds.), Explorations in the ethnography of speaking (pp. 433-451). Cambridge: Cambridge University Press.

- (1992). «The concept of communicative competence revisited», Thirty years of linguistic evolution, pp. 31-57.

Instituto Cervantes (2006). Plan curricular del Instituto Cervantes. Niveles de referencia para el español. Madrid: Instituto Cervantes-Biblioteca nueva.

KUZNETSOV, G. (2010). "Algunas particularidades socioculturales y lingüísticas del proceso de enseñanza - aprendizaje de ELE a inmigrantes rusohablantes en España», Marco ELE, 11, pp. 1-14.

Ley orgánica para la mejora de la calidad educativa (LOMCE) (Ley Orgánica 8/2013, 9 de diciembre). Boletín Oficial del Estado, 295, 2013, 10 diciembre.

MAATI BEGHADID, H. (2013). «El enfoque comunicativo, una mejor guía para la práctica docente», Actas del IV taller ELE e interculturalidad, Instituto Cervantes de Orán, pp. 112-120.

Morales Orozco, L. (2006). La integración lingüistica del alumnado inmigrante: propuestas para el aprendizaje cooperativo. Catarata: España.

Márquez VÁzquez, C. y Gualda CABALlero, E. (2013/2014). «Absentismo escolar en secundaria: diferencias entre nacionales e inmigrantes en la provincia de Huelva», En clave Pedagógica, n. ${ }^{\circ} 13$.

NunAN, D. (1989). El diseño de tareas para la clase comunicativa. Cambridge: Cambridge University Press. 
RoCA MARÍN, S. (2002a). «Esbozo de un modelo de organización de ELE en Educación Secundaria Obligatoria», I Encuentro nacional de atención a la diversidad: educación e inmigración, pp. 1-5.

- (2002b). «Materiales de E/LE en educación secundaria obligatoria», Estudios de Lingüística, n. ${ }^{\circ} 16$, pp. 5-15. 